CONF-9604125--2

UCRL-JC-123503

PREPRINT

\title{
Partial Discharge Characterization of High-Voltage Cables and Components
}

\author{
R. L. Druce, and R. S. Lee \\ Lawrence Livermore National Laboratory
}

This paper was prepared for submission to the

20th Compatibility, Aging, and Stockpile

Stewardship Conference

Kansas City, MO

April 30-May 2, 1996

February 28, 1996

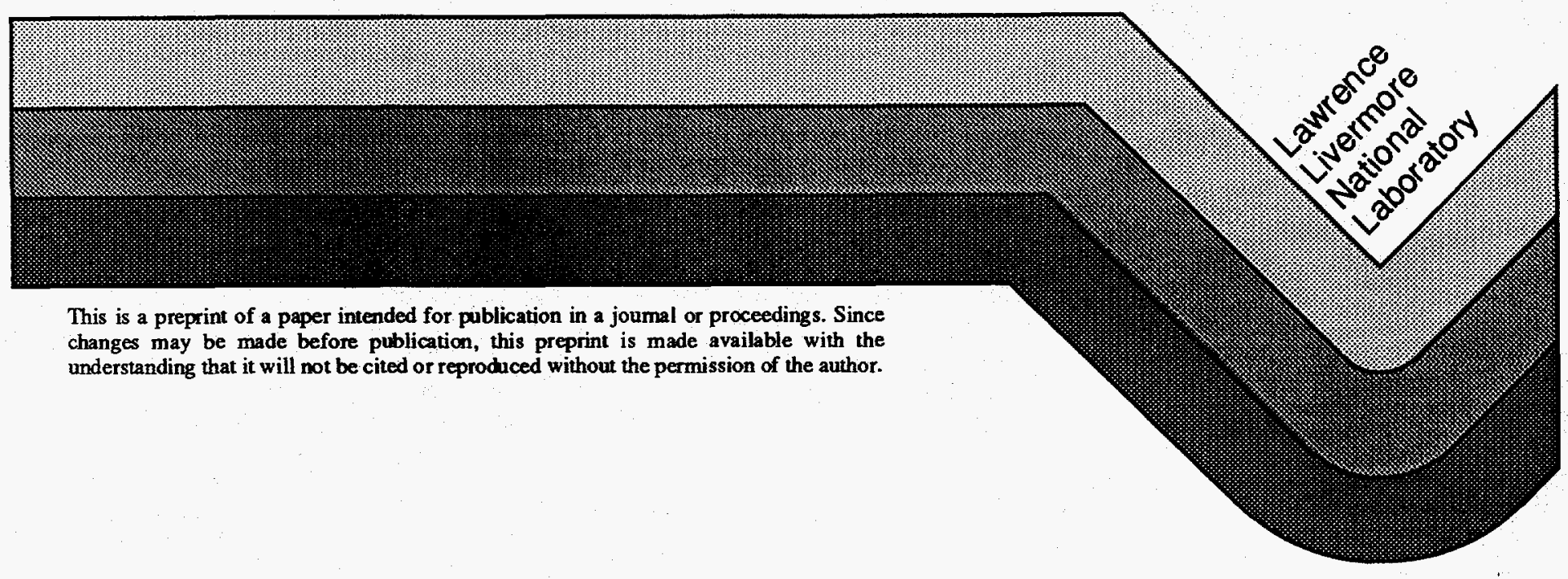




\section{DISCLAIMER}

This document was prepared as an account of work sponsored by an agency of the United States Government. Neither the United States Government nor the University of California nor any of their employees, makes any warranty, express or implied, or assumes any legal liability or responsibility for the accuracy, completeness, or usefulness of any information, apparatus, product, or process disclosed, or represents that its use would not infringe privately owned rights. Reference herein to any specific commercial products, process, or service by trade name, trademark, manufacturer, or otherwise, does not necessarily constitute or imply its endorsement, recommendation, or favoring by the United States Government or the University of California. The views and opinions of authors expressed herein do not necessarily state or reilect those of the United States Government or the University of Califomia, and shall not be used for advertising or product endorsement purposes. 


\title{
Partial Discharge Characterization of High-Voltage Cables and Components*
}

\author{
R. L. Druce and R. S. Lee \\ Lawrence Livermore National Laboratory \\ Livermore, CA 94550
}

Introduction: The goal of this work is to predict the dielectric strength and lifetime of detonator cables and other high-voltage components in the stockpile, to detect changes in dielectric response which are precursors of degradation of high-voltage standoff capability and lifetime, and to identify manufacturing techniques which can improve the performance of high-voltage cables and components. In pursuit of this goal, we plan to perform partial discharge (PD) testing on detonator cables and high voltage components. The study will involve developing a test protocol which allows us to predict degradation of dielectric materials based on non-destructive, PD tests. Tests will be performed on new cables to establish baseline behavior and then on cables recovered from stockpile to look for changes. The payoff for this work will be a quantitative, nondestructive test which relates to cable lifetime and high-voltage standoff for new and aged cables.

PD testing will also be useful for optimizing manufacturing processes to increase cable reliability and can be used to characterize other high-voltage components, such as connectors. In subsequent years we plan to study manufacturing processes and other components. Electromagnetic field models will be used to help understand the PD results and for optimization of geometrical factors.

Detonator Cable Testing: High-voltage components in a nuclear weapon must be tested for integrity before installation and, preferably, throughout the stockpile lifetime. Many of these components, for example the detonator cables, are critical because their failure can prevent the weapon from functioning. Destructive testing of unfinished cables typically consists of applying a steadily increasing high voltage across an open cable until the cable suffers catastrophic breakdown. The intrinsic high voltage breakdown level is determined by the type and condition of the dielectric and by the geometrical configuration of the cable conductors (neglecting end effects and connectors).

Nondestructive evaluation of detonator cables is performed before the detonator is installed and consists of applying a high voltage across a cable (greater than the operating voltage) for a specified period of time (hi-pot test). If there is no anomalous behavior, the cable is presumed to be acceptable. This tests has two shortcomings: there is no quantitative measure of the quality of the cable and there is a finite probability that the test could damage the cable sufficiently to cause failure when it is used to fire a detonator. Partial Discharge Testing: PD testing has the potential to provide both a quantitative statement of the quality of a cable and to virtually guarantee that any potential of damage will be detected by the test. PD testing detects transfer of charge or "micro breakdowns"

Work performed under the auspices of the U.S. Department of Energy by the Lawrence Livermore National Laboratory under Contract W-7405-Eng-48. 
which occur in the interior of a dielectric undergoing electric stress. This situation is illustrated schematically in Fig. 1. A dielectric specimen under test is represented as a

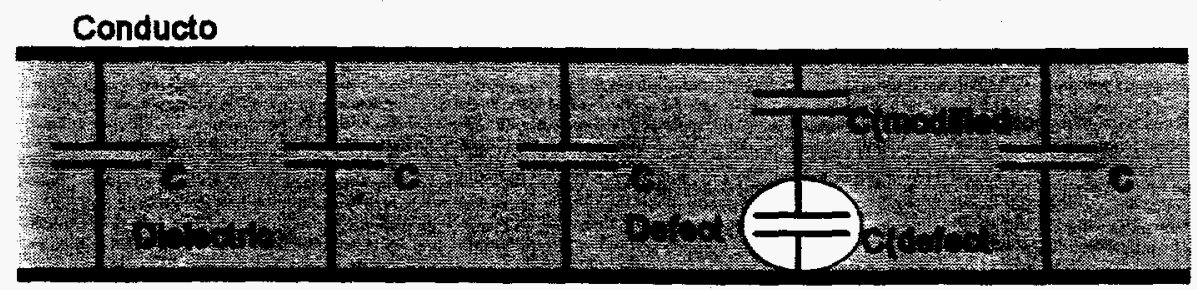

Figure 1. Electrical schematic of a dielectric with an internal defect.

parallel combination of capacitors. The section of the sample with the defect is represented as two capacitances in series. If charge transfer (breakdown) occurs within the defect, charge will be redistributed over the other capacitances, resulting in a current pulse which can be detected in an external circuit. The measuring circuit is shown schematically in Fig. 2.

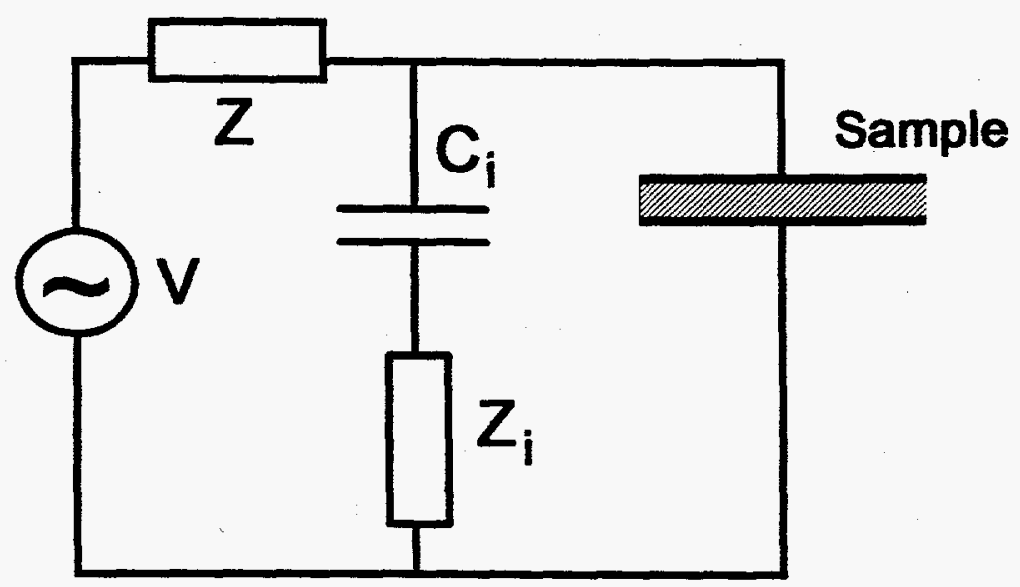

Figure 2. Schematic of a circuit for PD measurements. An alternating voltage is applied across the sample. $C_{i}$ and $Z_{i}$ represent the input impedance and input capacitance of the circuit which records the voltage across the sample.

To perform the measurement, the sample is connected to a voltage source as shown in Fig. 2. Discharge currents are seen as spikes which are superimposed on the sample voltage waveform, as is shown schematically in Fig. 3.

Several years ago there was a program at LLNL which included PD testing of detonator and distribution cables. This work showed that it is possible to relate PD signatures to ultimate cable failure and quality (1). Additionally, the work showed that geometry is an important factor in designing for reliability and that geometry and air voids produced in manufacture combine to affect reliability in a non-intuitive way. More recently, a French group (2) demonstrated a correlation of PD signatures with increased cable life produced by heat treatment during manufacture and with reduced cable life caused by harsh environments. 


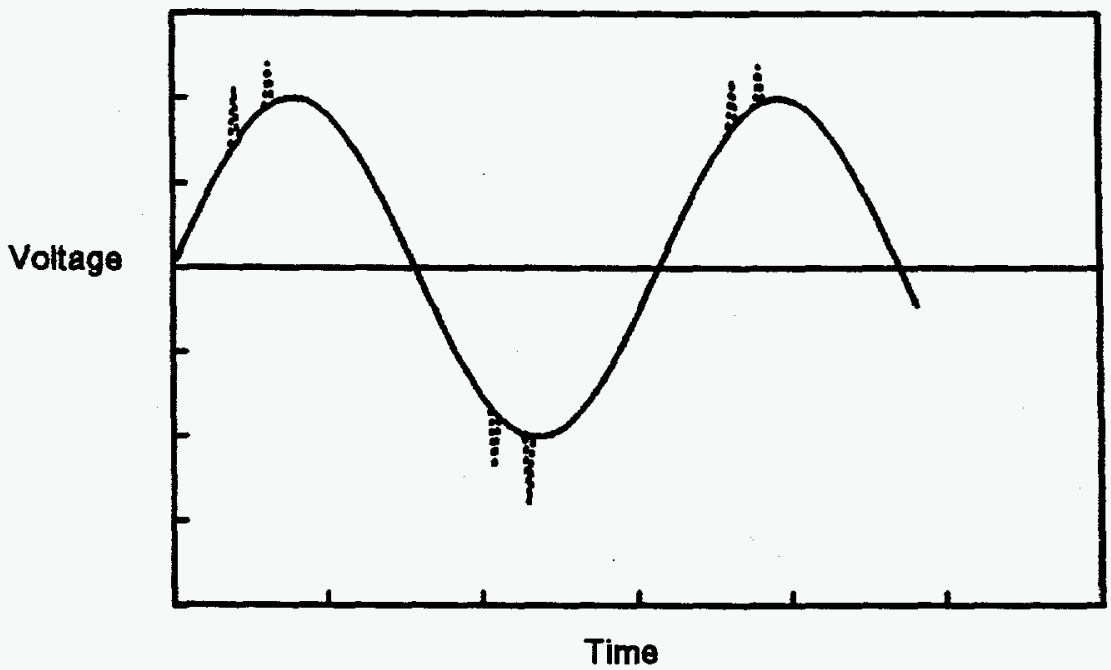

Figure 3. Schematic representation of partial discharge events superimposed on the sample voltage waveform.

Methodology: Attaining full confidence in the predictive capability of PD testing for detonator cables will take three to five years. We plan to begin the work this year by testing several new cables of different manufacture. Continued work will test cables aged artificially and naturally in subsequent years. Following the lead of past studies $(1,2)$ we will develop a testing protocol which will include destructive and PD testing on lots of cables of different manufacture. The PD tests will be correlated with destructive tests to develop confidence in the predictive capability of the PD technique. We will also use destructive tests to age cables to near destruction and monitor the PD signatures to note the effect of repeated stress on cables. We will begin this year's work using existing equipment housed in Building 141 at LLNL (Pulsed Power Lab). We will work with members of the Electromagnetics and Diagnostics Group to understand the capabilities of the present equipment and setting up the initial experiments and in the interpretation of the initial results.

Future Work: At the conclusion of this year's work we will be ready to test cables that have been subjected to aging factors. We also plan to procure a portable PD system that can be used in conjunction with high explosive firing tanks to investigate structures that contain small quantities of high explosive (detonators).

\section{References:}

1. L. B. Gordon, R. L. Druce and M. J. Wilson, Failure Modes of Laminate Structures, Sixth IEEE Pulsed Power Conference Proceedings, Arlington, VA (1987).

2. R. Boucheteau, H. Biero and C. Prisset, Charactérisation de composants haute tension par décharges partielles, Proceedings of 6th International Congress on Pyrotechnics, Tours, France (1995). 